\title{
DEVELOPMENT AND VALIDATION OF A PFA MODEL FOR COMPOSITE AERONAUTICAL STRUCTURES WITH STRESS CONCENTRATORS
}

\author{
M. V. ANGELO ${ }^{123}$, A. C. GALUCIO ${ }^{1}$, V. TITA ${ }^{3}$ \\ ${ }^{1}$ EADS Innovation Works, Paris, France. (marcus.angelo@ helibras.com.br) \\ ${ }^{2}$ Helicópteros do Brasil - Helibras, Itajubá, Brazil \\ ${ }^{3}$ Engineering School of São Carlos - University of São Paulo, São Carlos, Brazil
}

Besides advances on failure of composite materials accomplished lately, problems involving damage tolerance are still a field wide open for researches. The state of art theory is still complex and hard to be applied widely in industry level. This work aims to provide a computational tool to calculate composite aeronautical structures such as fuselage and rotor blade considering large damage capabilities. The computational tool is based on the theories proposed by Puck and Matzenmiller, which predict damage propagation under a Progressive Failure approach. It is important to note that even on a mesoscale level, the subroutines separate the mechanisms concerning the fiber and the matrix (inter-fiber) and treat them under different perspectives. Material elastic and ultimate properties are obtained from standardized experimental tests, while for specific parameters, new procedures have been developed. From common tension and compression $[0]_{n},[ \pm 45]_{n},[ \pm 67.5]_{n}$ tests, a package of subroutines analyzes raw experimental data providing these parameters. Additionally, numerical models are calibrated and literature data are adopted. Calculus kernel is coded in FORTRAN (UMAT/URDFIL) and linked to Abaqus ${ }^{\circledR}$. A high order plane stress material model is employed and different governing laws are applied for fiber and inter-fiber damage. Also, a non-local criterion is used to provide spatial regularization and thus avoid convergence numerical problems. For evaluating consistency at coupon and structural element level, a series of simulations is carried out. Material characterization is checked against experiments on low scale elementary coupons, while implementation is verified by simulating structures in element level. A detailed study of mesh and step size influence is also performed. Finally, validation is held against tests of open-holed and notched plates. It can be seen that the ultimate load is correctly predicted as well as the stiffness during loading. Besides, strain gage response, load cell results and x-ray maps are compared with simulation data. The comparison of results shows that the proposed computational tool can predict the behavior of large damaged composite structures. As future work, a subroutine to analyze delamination can be implemented to improve the numerical predictions performance, for turning this computational tool into a real industry-level virtual testing utility.

Keywords: Composite Structures, Progressive Failure Analysis, Non-Local Criterion 


\title{
NOTATION:
}

\author{
$S_{12} \quad$ Shear Strength \\ $Y^{t} \quad$ Transversal Strength \\ $p_{\| \perp}^{t} \quad$ Puck's Inclination Parameter \\ $I F F_{A} \quad$ Failure Exposure Index \\ $\eta_{E / G} \quad$ Damage Variable for Transverse and Shear \\ $\eta_{r}^{E / G} \quad$ Friction Parameter - Residual Factor \\ $c_{E / G} \quad$ Puck's Parameter \\ $\xi_{E / G} \quad$ Puck's Parameter \\ $G_{12} \quad$ Corrected Shear Modulus \\ $G_{12}^{0} \quad$ Original Undegraded Shear Modulus \\ $E_{22}$ Corrected Transversal Modulus \\ $E_{22}^{0} \quad$ Original Undegraded Transversal Modulus \\ $\gamma_{12} \quad$ Shear Strain \\ $\zeta \quad$ Fiber Non-Linearity Coefficient \\ $C_{i} \quad$ Shear Non-Linearity Coefficient \\ $\overline{\varepsilon_{11}} \quad$ Regularized Longitudinal Strain \\ $\varepsilon_{11}^{i} \quad$ Longitudinal Strain in i-th Integration Point \\ $V^{i} \quad$ Characteristic Volume \\ $\varepsilon_{t / c}^{f} \quad$ Strain at Failure on $0^{\circ}$ Test \\ $\omega \quad$ Damage Variable for Longitudinal \\ $m_{t / c} \quad$ Matzenmiller's Parameter \\ $e_{t / c} \quad$ Matzenmiller's Parameter \\ $E_{11} \quad$ Corrected Longitudinal Modulus \\ $E_{11}^{0} \quad$ Original Undegraded Longitudinal Modulus
}

\section{INTRODUCTION}

Even though huge advance on the analysis of the failure on composite materials was accomplished in later years, the problem involving the concept of damage tolerance in such structures is still a field wide open for researches. The state of art theory, the fracture mechanics approach, is still too complex and hard to be applied widely in an industry level. The aim of this work consists on providing a complete computational tool to calculate, regarding large damage capabilities, composite aeronautical structures, such as fuselages and rotor blades for instance. 
The current work presents a computational tool, which contains a model based on the theory of Puck and Matzenmiller and calculates the damage propagation on a composite material on a Progressive Failure Analysis (PFA). Also, a utility for automatically identifying related parameters from raw test data was developed. The core routine was coded in an Abaqus $^{\circledR}$ UMAT/URDFIL (1) routines and aims to reproduce structure behavior, once the applied load starts to damage it and the mechanical properties are reduced.

Analysis is sub-divided in prior and after failure threshold. In the first phase an update of mechanical properties is made to proper reproduce observed non-linearities in this phase. In the second step, once failure has established, damage variables are calculated to quantify the damage in each point of calculus. This model was one of the best evaluated in Worldwide Failure Exercise I and showed good results in WWFEII. "Exhibiting good predictive capability, none or one fundamental weakness and many relatively minor weaknesses" (2)

The target structures are large composite plates with stress concentrator simulating, for instance, a fuselage panel cut by impact. The validation was made against elements of notched and open-holed plates (Figure 1). The routine adopts plane stress constitutive law (Middlin-Reissner) which allows prediction of intra-ply failure, with an estimation of out of plane efforts, over shell S4R elements as presented in (3). Therefore, the constitutive equations are represented in equation (1):

$$
\left\{\begin{array}{l}
\sigma_{x x} \\
\sigma_{y y} \\
\sigma_{y z} \\
\sigma_{x z} \\
\sigma_{x y}
\end{array}\right\}=\left[\begin{array}{ccccc}
\overline{Q_{11}} & \overline{Q_{12}} & 0 & 0 & \overline{Q_{16}} \\
\overline{Q_{11}} & \overline{Q_{11}} & 0 & \overline{0} & \overline{Q_{26}} \\
0 & 0 & \overline{Q_{44}} & \overline{Q_{45}} & 0 \\
\overline{0} & \overline{0} & \overline{Q_{45}} & \overline{Q_{55}} & \overline{0} \\
\overline{Q_{16}} & \overline{Q_{26}} & 0 & 0 & \overline{Q_{33}}
\end{array}\right]\left\{\begin{array}{c}
\varepsilon_{x x} \\
\varepsilon_{y y} \\
\varepsilon_{y z} \\
\varepsilon_{x z} \\
\varepsilon_{x y}
\end{array}\right\}
$$

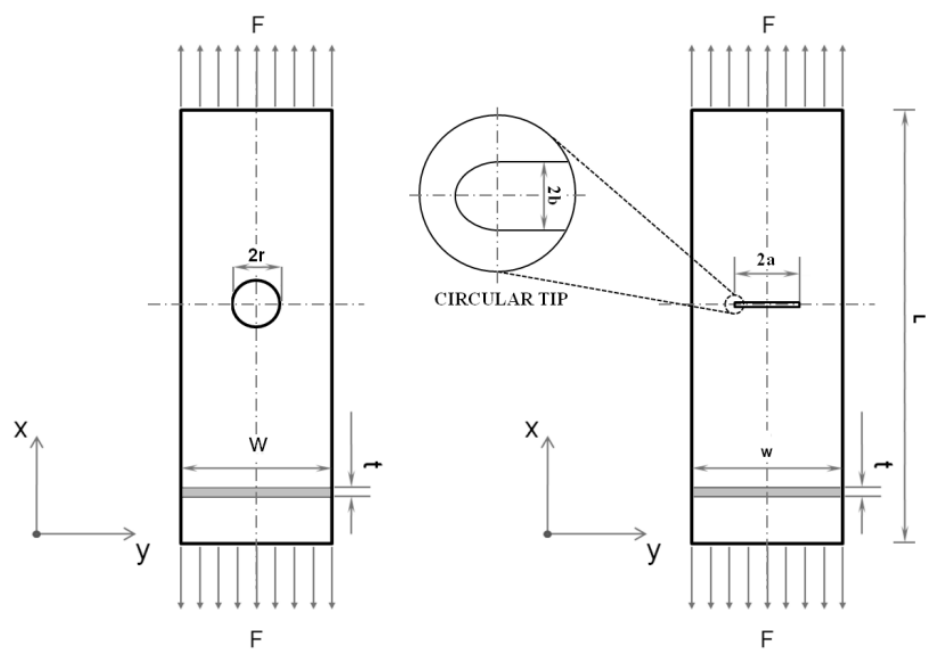

Figure 1. Composite structures investigated in this work.

\section{METHODS}

Despite the fact that it acts on a mesoscale level, the routine separates the failure mechanisms into the ones concerning the fiber and the ones concerning matrix (inter-fiber) 
and approaches each of them under a different perspective.

Matrix mechanisms of damage are of great interest in high-performance industries as they might be present even in a still non-collapsed structure. In order to correct modeling it, the present job invokes Mohr's assumption. According to it the collapse of the matrix is dependent on the stress state acting on the plane in which failure occurs.

On the other hand, fiber mechanisms are very brittle and abrupt. Since the fibers are the main responsible for providing stiffness in a composite structure, their failure strongly modifies the stress/strain field and quickly reduces its strength, which implies concentration and convergence problems on a FEA (Finite Element Analysis).

To proper reproduce both of them, Matzenmiller's theory (4) was applied for mechanisms in the longitudinal direction and Puck's theory (5) in the transverse and shear directions. Also, a non-local approach was adopted to avoid a premature cut in calculus due to convergence problems, when fiber-failure establishes, as described at (6).

As a common procedure in structural analysis, the determination of all required parameters precedes the calculus itself. For the presented model, most of the input data is already taken from ordinary tests. For example, mechanical properties, maximum strains and stresses in rupture can be obtained from normalized and standardized experiments. Regarding the model constants, the acquisition had no protocol, methods or procedures of identification. Hence, they were developed and their influences in the results were deeply considered. Efforts were made to reduce the amount of tests required by using common experiments for acquisition and also taking as reference values of constants to which the model is very insensitive.

With results from basic specimens $\left[0^{\circ}\right]_{\mathrm{n}},\left[ \pm 45^{\circ}\right]_{\mathrm{n}},\left[67.5^{\circ}\right]_{\mathrm{n}}$, under tension and compression, a package of sub-routines was written to treat raw test data and provide the parameters required to run the model. For parameters, which could not be extracted from basic tests, a model calibration analysis was made. Finally, some parameters were taken from literature (7) when identification and/or calibration were not possible.

Concerning the code itself, the routine runs a finite element model at Abaqus ${ }^{\circledR}$ with UMAT/URDFIL, according to the sequence shown by Figure 2 .

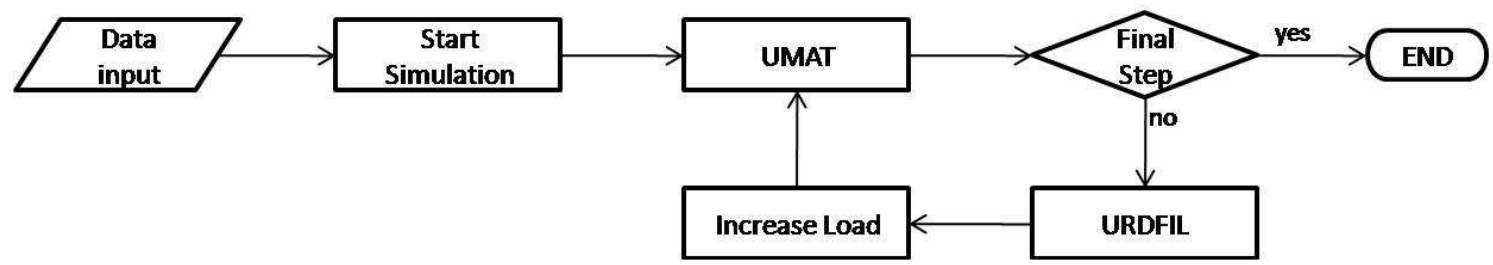

Figure 2. Routine Flux: Computational Tool.

Concerning Figure 2, for each step n, the "virtual" stress-strain state of the structure is calculated, using the mechanical properties from step n-1. Middlin-Reissner, as presented in (8), theory for composite plates is used with a second degree law for longitudinal direction and third degree for transverse shear strains.

$$
E_{11}^{t / c}=E_{11}^{0}\left(1+\zeta^{t / c} \varepsilon_{11}\right) \quad G_{12}=C_{3} \gamma_{12}^{2}+C_{2} \gamma_{12}+C_{1}
$$


This virtual state will be used to quantify the amount of damage each element. At this point, fiber and inter-fiber mechanisms should be treated separately (9), i.e., there is not considered coupling effects.

For the matrix failure (inter-fiber failure), a new coordinate system is defined at each mesh integration point, according to Puck's "slope" and the "action plane" in which the stresses are acting. Three modes of failures are defined concerning the sign and ratio between components of the stress tensor and each mode has its own governing law and set of parameters. They are remarkably different from classic crack modes from Theory of Fracture Mechanics (10). By averaging the stress tensor in the new orientation system (as described by Puck's Theory), a failure index is calculated according to Equation (3) based on the so-called "stress exposure" and used to quantify the amount of damage in the matrix at each integration point. This index is passed as argument along with the ultimate strengths for the Equation (4), which calculates the damage variables of the inter-fiber mechanism.

$$
\begin{gathered}
I F F_{A}=\sqrt{\left[\left(\frac{1}{Y^{t}}-\frac{p_{\perp \|}^{t}}{S_{12}}\right) \sigma_{22}\right]^{2}+\left(\frac{\sigma_{12}}{S_{12}}\right)^{2}}+\frac{p_{\perp \|}^{t}}{S_{12}} \sigma_{22} \\
\eta_{E / G}=\frac{1-\eta_{r}^{E / G *}}{1+c_{E / G}^{*}\left(I F F_{A}-1\right)^{\xi_{E / G}^{*}}}+\eta_{r}^{E / G *}
\end{gathered}
$$

The shear and transverse damage variables are then used to upgrade the material properties $\mathrm{E}_{22}$ and $\mathrm{G}_{12}$. Equation (5) shows the degradation of the properties due to matrix failure:

$$
E_{22}=\eta_{E} E_{22} \quad G_{12}=\eta_{G} G_{12}
$$

For the fiber failure, the brittle and abrupt character is treated with a non-local approach. For each integration point in the mesh, a characteristic neighborhood is defined due to a non-local criterion as described by (6). It contains the set, herein called characteristic volume of rupture (CVR) of integration points within a distance of the characteristic radius (CR) Figure 3.

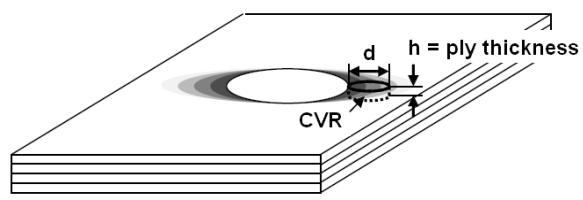

Figure 3. Characteristic Volume

The value of the $\mathrm{CR}$ is a material characteristic and may be obtained from elementary tests with stress concentrator presence. All the neighborhoods are defined in the first call to the URDFIL routine (Figure 2). At each subsequent step, after the current virtual stress-strain state is calculated, URDFIL makes a sweep throughout the structure and, for each point, calculates a weighted average of the current longitudinal strain within the region bounded by 
each set as displayed by Equation (6).

$$
\overline{\varepsilon_{11}}=\frac{\sum^{i} \varepsilon_{11}^{i} V^{i}}{\sum^{i} V^{i}}
$$

This procedure intends to minimize concentration, which may affect the convergence during the calculus and it is a space criterion to control high strain rates and gradients. The averaged strain is passed as argument along with mechanical ultimate longitudinal strain to Equation (7), which calculates the longitudinal damage variable to be used for updating $\mathrm{E}_{11}$.

$$
\omega=1-\exp \left(-\frac{1}{m_{t / c}^{*} e_{t / c}^{*}}\left(\frac{\varepsilon_{11}}{\varepsilon_{t / c}^{f}}\right)^{m_{t / c}^{*}}\right)
$$

The longitudinal damage variable is then used to upgrade the material properties E11. Therefore, the Equation (8) shows the degradation of the property due to fiber failure:

$$
E_{11}=\omega E_{11}^{0}
$$

It is important to mention that all variables signed with * (in the equations above) are parameters of the model. Once the mechanical properties have all been updated, the new "real" stress-strain state of the structure is calculated (i.e. the constitutive equations are updated) for the step of solution and the related variables are stored and passed for input data into the calculations of the next step of solution.

A deep verification was performed for different model levels, using the computational tool implemented. Parametric identification and calibration were compared to results of elementary specimen tests. Numeric simulation results were compared experimental data in order to verify the accuracy of proposed methods for determination of the whole set of parameters involved.

After the identification and calibration process of parameters, PFA was carried out to predict the mechanical behavior of simple composite structures. Thus, computing issues and each module performance were verified by running elements, which represents the interest structures like notched and open-holed single or multi oriented. In order to certify a trustful result, studies of mesh and step size influence over the model were conducted, also. Due to the intrinsic characteristics of the models, mesh sizing must be carefully carried to assure that stress concentration zones will have enough integration points inside each neighborhood (for non-local criterion) such as the analysis does not abort prematurely. On the other hand, step sizing is important since it defines the "virtual" stress strain state, which will be used to quantify the damage variables. Thus, a smooth propagation of the damage process is absolutely necessary to achieve good results. A sensibility analysis was made to evaluate the influence they have over the structure response. A series of analysis was carried out varying one parameter at a time. Tendencies of response are displayed in Figure 4. It was defined an "acceptable zone" in each case. This zone, shown in grey, represents the range of validity established for the respective parameter. A proper choice of these factor has great impact in 
calculus time since it is possible to define, compromise-wise optimal values for mesh sizing and step sizing for instance. The effectiveness of the applied non-local criterion can easily be noticed by the low influence that mesh size has over the response. Due to confidentiality of data, every result hereafter was normalized, so that maximum values in the range considered in the axis are taken as " 1 ".

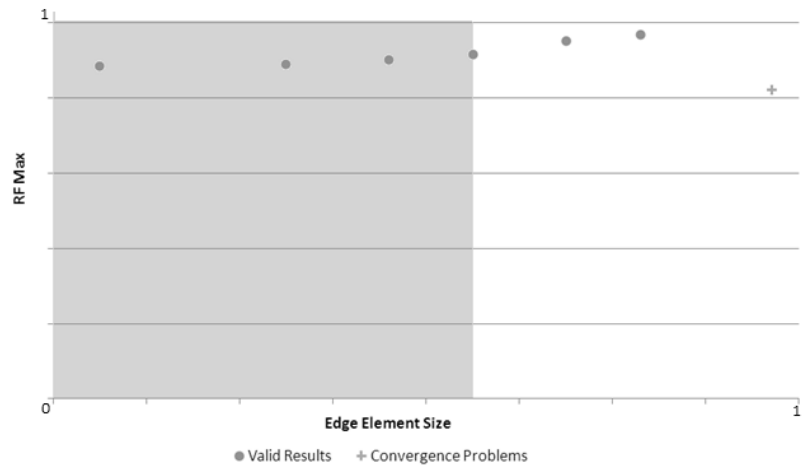

(a)

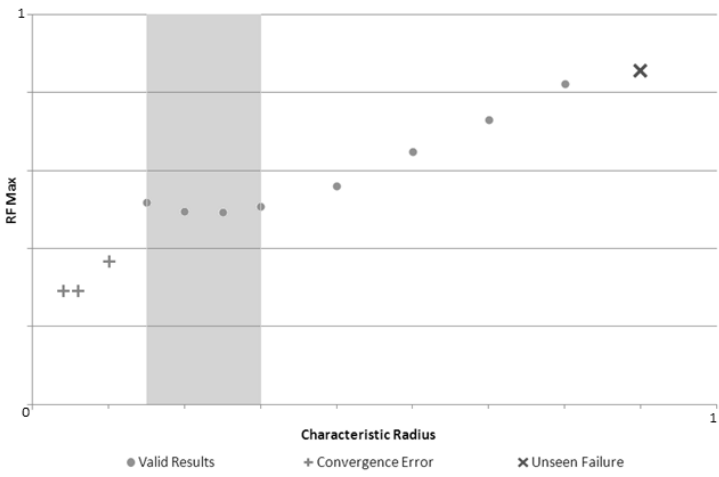

(b)

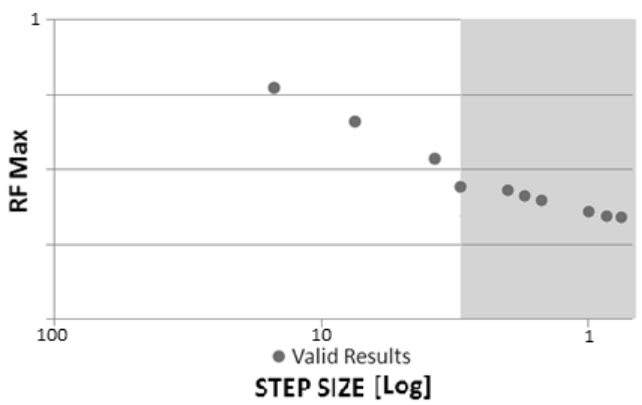

(c)

Figure 4. Maximum Reaction Force (RF Max) sensibility analysis concerning: (a) Mesh Size (b) Characteristic Radius (c) Step Size.

\section{RESULTS}

The computational tool was applied to analyze open holed and notched plates as shown by Figure 1. Table 1 displays the geometry and the stacking sequence of the analyzed composite structures. It is important to highlight the dimensions of the composite structures. In fact, a total of 5 specimens were investigated with the specifications described at Table 1.

Table 1. Geometry ${ }^{*}$ and stacking sequence of the analyzed composite structures.

\begin{tabular}{cccc}
\hline Family & 1 & 2 & 3 \\
\hline Type & Circular Opening & Notch & Notch \\
\hline $\mathrm{L}$ & 75 & 900 & 150 \\
\hline $\mathrm{w}$ & 22.5 & 300 & 90 \\
\hline $\mathrm{t}$ & 1 & 1 & 1 \\
\hline $\mathrm{r}$ & 3.25 & - & - \\
\hline $\mathrm{a}$ & - & 25 & 7.5 \\
\hline $\mathrm{b}$ & - & 0.25 & 0.25 \\
\hline \multirow{2}{*}{ Stack } & {$\left[45^{\circ}, 0^{\circ},-45^{\circ}, 90^{\circ}\right]_{2 \mathrm{~S}}$} & {$\left[45^{\circ}, 90^{\circ}, 45^{\circ},-45^{\circ}, 45^{\circ}, 0^{\circ}\right.$,} & {$\left[90^{\circ},-45^{\circ}, 90^{\circ}, 0^{\circ}, 90^{\circ}, 45^{\circ}\right.$,} \\
\hline
\end{tabular}

(*) normalized by thickness 
The strain value in direction $x$ was acquired via strain gages (aligned with $x$ - Figure 1) for notched specimens or by Digital Image Correlation for the open hole. The loading was collected by an appropriate load cell. Thus, the experimental results for force $v s$. strain or displacement are compared to numerical analyses with prescribed displacement.

Considering the results shown by the Figure 4, in most cases, the ultimate load was well predicted and so was the stiffness. As it can be seen, the result provided by the computational tool agrees with experimental test results in spite of an overestimation of the maximum load in one case. Nevertheless, failure phenomena are always captured.

(a)

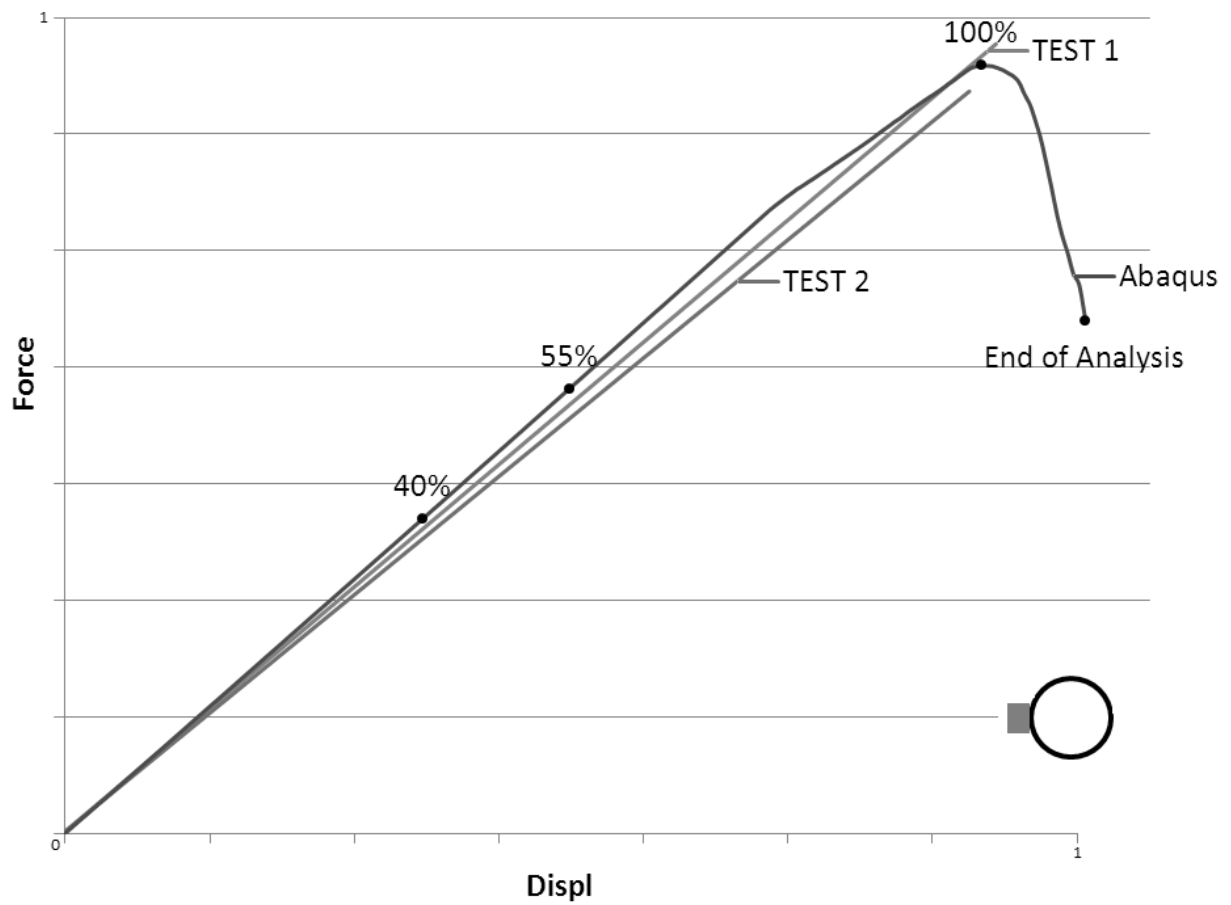

(b)

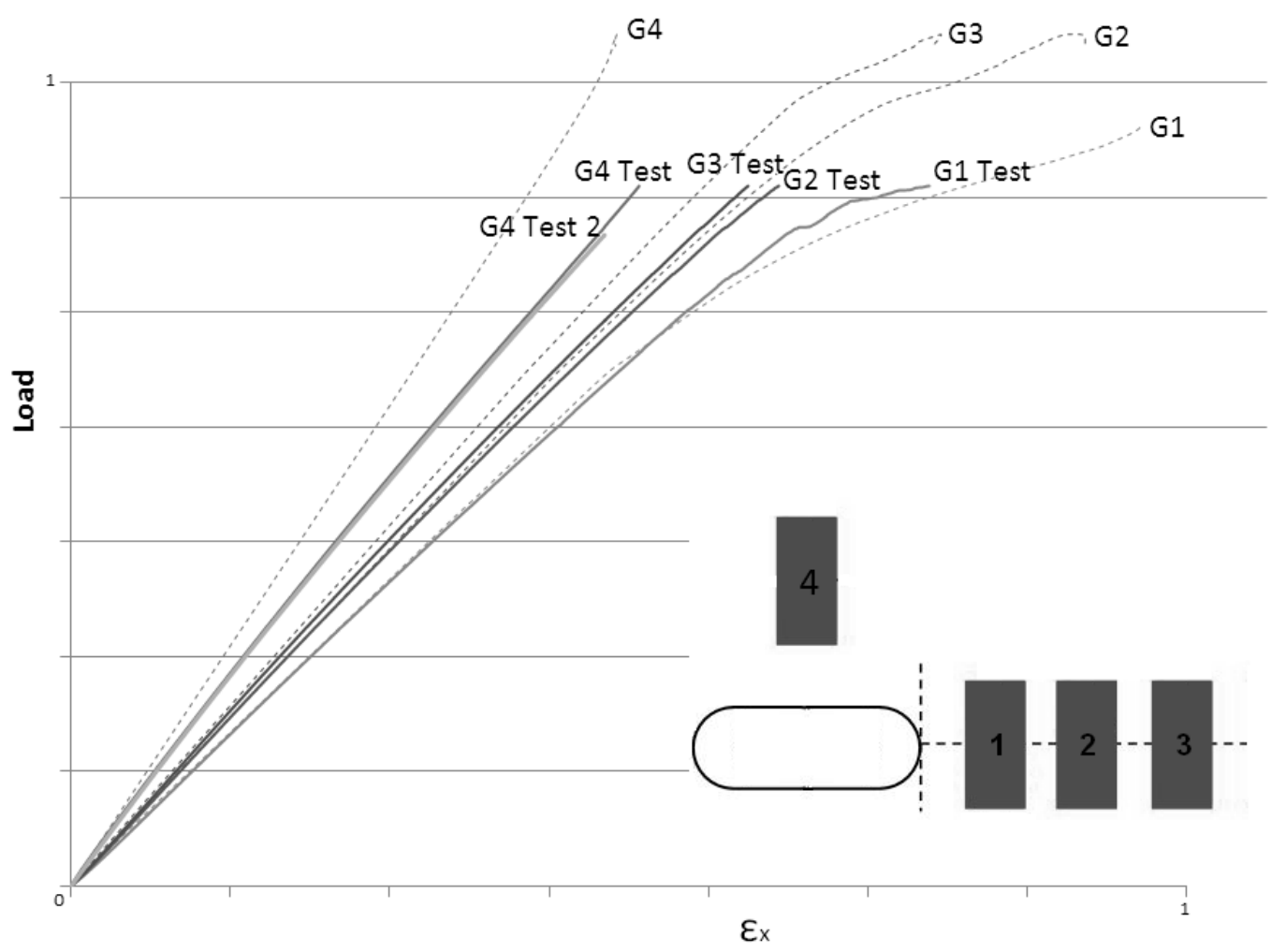


(c)

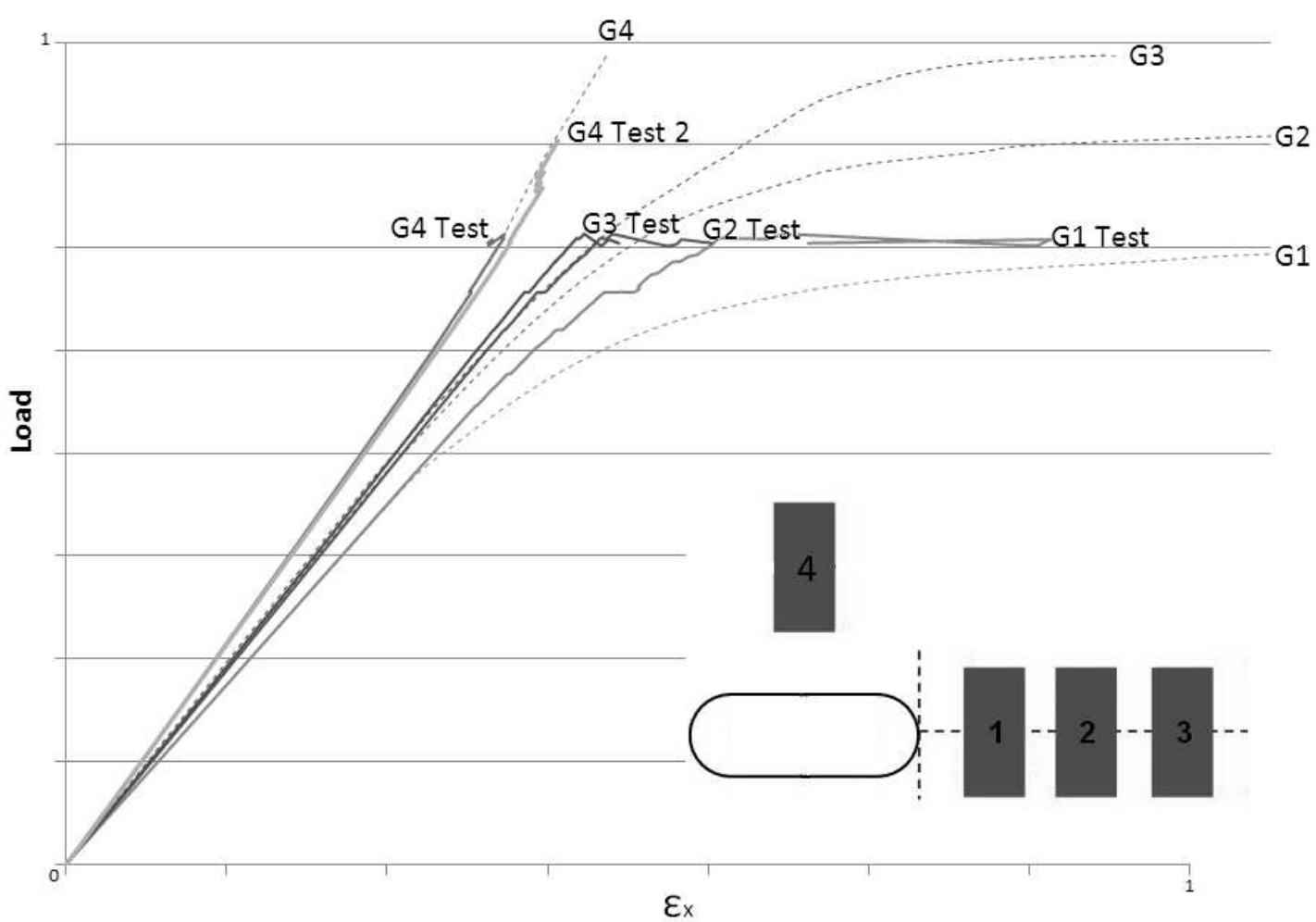

Figure 5. (a) Family 1: Force vs. Longitudinal Displacement; (b) Family 2: Force vs. Longitudinal Strain; (c) Family 3: Force vs. Longitudinal Strain

Finally, FEA damage maps were analyzed and compared to X-Rays analyses like shown by Figure 5. It is important to observe that the X-Ray image (Figure 5a) not only shows intra-ply failures but also delaminations,

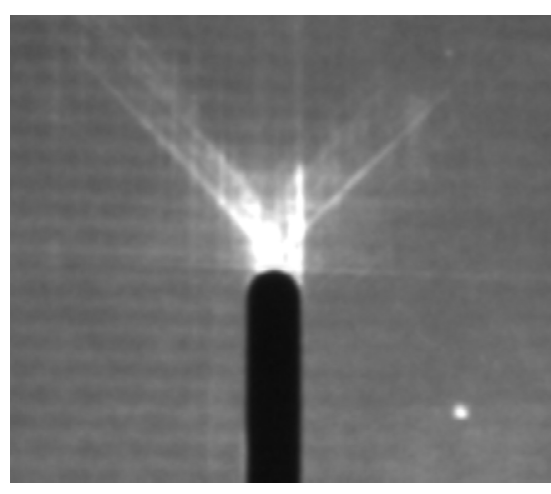

(a)

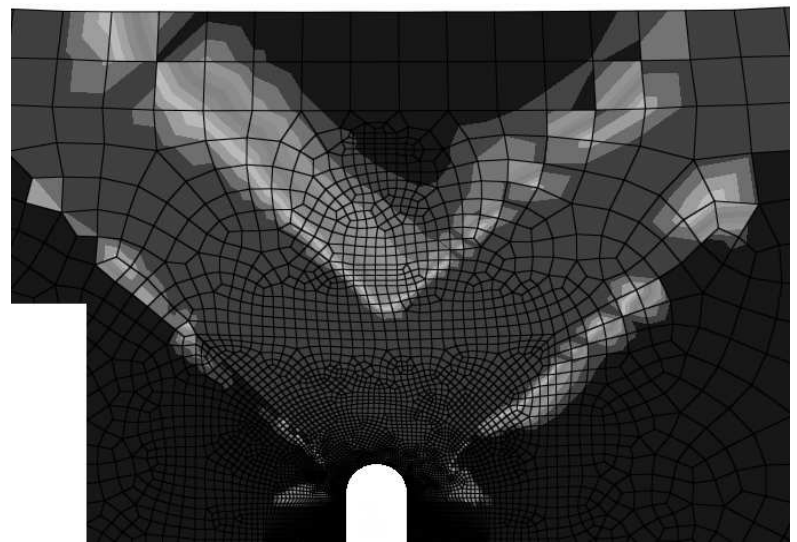

(b)

Figure 6. Results for Family 3: (a) X-Ray analysis (89\% of Maximum Load); (b) Matrix damage map provided by FEA (at the same load) 


\section{REFERENCES}

1. Abaqus. Abaqus Version 6.11 Documentation. 2011.

2. Hinton, M. J., Kaddour, A. S. et Soden, P. D. "A Comparison of the Predictive Capabilities of Current Failure Theories for Composite Laminates, Judged Against Experimental Evidence". Composite Science and Technology. 2002, Vol. 62.

3. Angélico, R. A. "Evaluation of Progressive Failure Models for Composite Material Structures”. s.1. : Master Thesis, School of Engineering of São Carlos, 2009.

4. Matzenmiller, A., Lubliner, J. et Taylor, R. L. "A Constitutive Model for Anisotropic Damage in Fiber-Composites". Mechanics of Materials. 20, 1995.

5. Knops, M. Analysis of Failure in Polymer Laminates: the Theory of Alfred Puck. s.l. : Springer, 2008.

6. Miot, S., Hochard, C. et Lahellec, N. "A Non Local Criterion for Modelling Unbalanced Woven Ply Laminates with Stress Concentrations”. Composite Structures. 2010.

7. Puck, A., Kopp, J. et Knops, M. "Guidelines for the Determination of the Parameters in Puck's Action Plane Strength Criterion”. Composites Science and Technology. 2002, Vol. 62. 8. Reddy, J. N. "Mechanics of Laminated Composite Plates and Shells - Theory and Analysis”. s.1. : CRC PRESS, 2003.

9. Deuschle, H. M. “3D Failure Analysis of UD Fibre Reinforced Composites: Puck's Theory Within FEA”. s.1. : PhD Thesis, Universitat Stuttgart. Vol. 92. 2010.

10. Puck, A. et Shürmann, H. Failure Analysis of FRP Laminates by Means of Phisically Based Phenomenological Models. Composite Science and Technology. Vol. 62, 2002. 